\title{
Psicologia do Desenvolvimento: Há Questões Novas?
}

\author{
Ana Maria Almeida Carvalho,", \\ ${ }^{1}$ Universidade de São Paulo - Instituto de Psicologia (IPUSP), São Paulo, SP, Brasil
}

\section{Resumo}

O objetivo deste texto é contribuir para o questionamento de certas concepções sobre a infância e o sentido do estudo do desenvolvimento. Recorrendo ao trabalho de Evelyn Thoman que, já na década de 80, examinou e questionou alguns pressupostos, em suas palavras, "mitos", que orientam a Psicologia do Desenvolvimento - os mitos do neonato deficiente, das competências aditivas, da neutralidade emocional e do futurismo - refletimos sobre sua evolução e recorrência na atualidade e apontamos alguns caminhos que temos seguido em nosso trabalho e que consideramos promissores para a crítica desses pressupostos e para a redefinição de conceitos como interação social, sociabilidade, seletividade, vínculo e cultura de pares.

Palavras-chave: Psicologia do desenvolvimento, infância, interação social, sociabilidade, cultura e brincadeira.

\section{Developmental Psychology: Are there New Issues?}

\begin{abstract}
This paper aims to contribute to a critical analysis of some notions about childhood and the meaning of the study of development. Resorting to the work of Evelyn Thoman who, back in the eighties, has already examined and questioned some assumed notions, in her words, "myths", that guide Developmental Psychology - the myths of the deficient neonate, of the additive competencies, of the infants' emotional neutrality and of futurism - we reflect about their evolution and recurrence in the contemporary research and point out some directions that we have explored in our work and that we consider as promising for a criticism of those assumptions and for the redefinition of concepts such as social interaction, sociability, selectivity, bonds and peer culture.
\end{abstract}

Keywords: Developmental Psychology, childhood, social interaction, sociability, culture and play.

\footnotetext{
Correspondência: Rua Pascoal Vita 405, apt. 153, 05445-000, São Paulo - SP, Brasil. Fone: (11) 38159738. anacarva2016@gmail.com.

Breve Descrição das Condições Originais de Apresentação do Trabalho: Ao ser homenageada no X Congresso Brasileiro de Psicologia do Desenvolvimento, promovido pela Sociedade Brasileira de Psicologia do Desenvolvimento (SBPD), agradeci a homenagem recebida e falei sobre pressupostos que ainda persistem ao se estudar o desenvolvimento infantil. A palestra foi videogravada e apresentada na sessão de abertura do Congresso, uma vez que não pude comparecer. O evento foi realizado no Hangar Centro de Convenções na Amazônia, em Belém-PA, no período de 18 a 21 de novembro de 2015, sob os auspícios da Universidade Federal do Pará, com o tema central "Inovação Científica e Tecnológica e a Promoção do Desenvolvimento Humano.
} 


\section{Psicología del Desarrollo: ¿Hay Nuevos Problemas?}

\section{Resumen}

El artículo tiene como objetivo contribuir al análisis crítico de algunas concepciones sobre la infancia y el significado del Estudio del Desarrollo. Recurrimos al trabajo de Evelyn Thoman, que ya entonces examinaba y criticaba algunas suposiciones, em sus palabras, "mitos", que guían la Psicología del Desarrollo - la del bebé deficiente, la de las habilidades cumulativas, la de la neutralidad emocional y del futurismo - reflexionamos sobre su evolución y su recurrencia actual, indicando las direcciones que hemos seguido en nuestro trabajo y que juzgamos prometedoras para la crítica de tales suposiciones y para la reformulación de conceptos como interacción social, sociabilidad, selectividad, vínculos y cultura de compañeros.

Palabras clave: Psicología del desarrollo, infância, interacción social, sociabilidade, cultura y juego.

Quero, em primeiro lugar, agradecer à Sociedade Brasileira de Psicologia do Desenvolvimento (SBPD) e a todos os colegas envolvidos pelo convite e pela homenagem, expressar o quanto significam para mim e me desculpar por não poder estar compartilhando pessoalmente esse momento com vocês. Receber uma homenagem como esta implica um reconhecimento de que, em algum nível, meu trabalho e nossa convivência deixaram alguma marca, fizeram sentido, tiveram algum significado. Qual ou quais? Revendo meu percurso, penso que tentei, por um lado, contribuir para o questionamento de certas concepções sobre a infância e o sentido do estudo do desenvolvimento. Em uma palestra apresentada na reunião do Centro de Investigação sobre Desenvolvimento Humano e Educação Infantil (Cindedi), em Ribeirão Preto, em 2007, procurei sintetizar essas preocupações recorrendo ao trabalho de Evelyn Thoman (1979). Meu objetivo era retomar o exame de alguns dos pressupostos que orientaram a Psicologia do Desenvolvimento ao longo de sua história e refletir sobre sua evolução ou recorrência na atualidade. Dito de outra forma, perguntamonos: há novidades nos pressupostos orientadores da pesquisa sobre desenvolvimento? Há novas questões inspiradas por novas descobertas e concepções resultantes a respeito do processo de desenvolvimento, e especialmente da infância?

Às vésperas dos anos 80 , Thoman já tinha empreendido uma tarefa similar, ao sintetizar uma crítica ao que chama de mitos a respeito da infância. Embora aponte que descobertas científicas contribuem para essas concepções, a autora sustenta, ao mesmo tempo, que a sua manutenção ou transformação é fruto da interação entre essas descobertas e valores sociais e culturais prevalentes em cada sociedade e em cada momento histórico - entre os quais se incluem valores políticos, econômicos, morais, religiosos; e, poderíamos acrescentar, até mesmo o conservadorismo da própria ciência enquanto instituição. Daí sua natureza de "mitos", no sentido de "construção mental de algo idealizado, sem comprovação prática; ideia, estereótipo", "valor social ... questionável, porém decisivo para o comportamento dos grupos humanos em determinada época" ou ainda, "afirmação fantasiosa, inverídica que é disseminada ...” (Houaiss \& Villar, 2001).

Refletindo particularmente sobre a primeira infância, Thoman (1979) examina criticamente quatro itens dessa "mitologia": os mitos do neonato deficiente (o bebê é incompetente por ser incapaz de suprir suas necessidades básicas); das competências aditivas (um ser competente é constituído por um somatório de competências independentes entre si); da neutralidade emocional (o bebê não é passível de estados emocionais, apenas de conforto e desconforto) e do futurismo (a infância é apenas um período de promessa, um prelúdio da vida adulta). Embora os analise separadamente, é evidente que há relações entre eles e que, em alguma medida, 
todos decorrem de um olhar referenciado pelo que é um adulto, que conduz apenas a uma obviedade - uma criança não é um adulto. As implicações resultantes dessa obviedade podem caminhar em duas direções: já que não é, deve tornar-se, e é nisso que consiste o processo de desenvolvimento; ou, alternativamente, não o é por boas razões adaptativas, que permitem vê-la como um organismo adequado e competente em cada momento de sua história de vida.

Desde a segunda metade do século 20 , inclusive sob o impacto da teoria do apego e seus desenvolvimentos desde os anos 60/70, enormes avanços técnicos e metodológicos permitiram novas formas de consultar o bebê e aprender com ele sobre a natureza da infância, caminhando na segunda direção sugerida. Não cabe aqui rever em detalhes a extensa literatura que fundamenta a visão cada vez mais prevalente sobre o bebê como um organismo finamente ajustado para obter do mundo aquilo que é essencial para sua sobrevivência: o contato e a interação ativa com o Outro. Nas palavras de Bussab et al. (2007, p. 102), "chegamos ao mundo com um encontro marcado"; e para esse encontro a evolução nos preparou cuidadosamente, em termos de capacidades sensoriais, de preferências perceptivas e motivacionais, de responsividade diferencial a aspectos relevantes do ambiente, de capacidades expressivas e de vida emocional (por exemplo, Bergamasco,1997; Bergamasco \& Beraldo,1990; Carvalho, 1988a; Delevati \& Bergamasco, 1999; Seidl de Moura \& Ribas, 2004; Tudella et al., 2000 - para citar apenas alguns trabalhos em nosso próprio meio). A compreensão integral dessa concepção só é possível se a evolução biológica da díade mãefilho for entendida como a evolução de um sistema, ou uma coevolução: nas palavras de Carvalho (1983, p. 277), "o ambiente natural de um bebê é o corpo de sua mãe".

Diante desse conjunto maciço de evidências e de sua integração em uma nova visão sobre a primeira infância, houve aparentemente uma superação dos mitos relativos a limitações e incompetências ou deficiências do bebê, inclusive - e talvez principalmente - sua suposta carência de vida afetiva (mitos do neonato deficiente e da neutralidade emocional). Em algumas áreas, no entanto, ainda parecem existir resquícios desses mitos, apesar das mais de quatro décadas decorridas desde as primeiras tentativas críticas. Parece-nos que esses resquícios ocorrem principalmente em perspectivas teóricas que enfatizam a cognição como precursora das competências socioafetivas e a linguagem verbal como definidora de humanidade; que limitam a ludicidade a formas de brincar nas quais se evidencia claramente a capacidade de representação, tal como ocorre no faz de conta; ou ainda que compreendem o raciocínio abstrato e dedutivo como marca e meta terminal da cognição humana. No limite, esse tipo de concepção implica considerar a primeira infância (ou mesmo toda a infância) como uma fase de pré-humanidade ${ }^{1}$. Implica ainda uma visão parcelada ou aditiva das capacidades ou aspectos envolvidos no processo de desenvolvimento - motores, sensoperceptuais, motivacionais e afetivos, sociais, cognitivos como se a cognição independesse dos demais aspectos, e cada um destes independesse entre si (mito das competências aditivas).

A nosso ver, no entanto, o mito mais renitente é o do futurismo que, em última instância, resulta dos mitos anteriores. O mainstream da Psicologia do Desenvolvimento continua a se perguntar sobre "em que vai dar", que condições ou características em cada fase são preditivas de desempenhos adultos ou mais maduros, que contextos de vida ou que intervenções são mais favoráveis para a superação da imaturidade infantil.

Essa visão já tem sido questionada desde o artigo clássico de Bruner (1976), com argumentos e evidências que apontam as vantagens adaptativas da imaturidade na infância. Lordelo

\footnotetext{
Não é raro encontrar na literatura a noção de que o desenvolvimento é o processo de "tornarse humano" ou de "humanização" - em uma apropriação inteiramente indevida de termos que fazem sentido na filogênese do ser humano, mas não em sua ontogênese.
} 
et al. (2008), retomando Bruner e outros autores mais recentes, atualizam essa crítica em um tom quase de libelo, focalizando particularmente suas implicações em termos de práticas educacionais. Reveem o conceito de adaptação ontogenética expressão usada na psicologia evolucionista para descrever e explicar características psicológicas cuja funcionalidade é aparente em certas fases do desenvolvimento, e que se perdem ou se transformam em fases posteriores, dando lugar a novas formas de adaptação. Os vários reflexos, as competências e preferências sensoperceptuais típicas da primeira infância, a prevalência da motivação lúdica e da exploração, a imaturidade motora e cognitiva, e mesmo os medos infantis (que a psicanálise clássica classificou como neuróticos) são entendidos, à luz desse conceito e das evidências empíricas revistas pelas autoras, como funcionais e necessários para a qualidade de vida na infância e para a própria sobrevivência. As conotações negativas de termos como "infantil" e "imaturo", assim como sua contrapartida, a valoração positiva da precocidade, são alvos de uma crítica radical. Desconhecer "a natureza e os usos da imaturidade" (Bruner, 1976) e buscar cegamente sua superação, e quanto mais cedo melhor, revelam ignorância e incompreensão sobre a natureza da infância e evidenciam a permanência do mito do futurismo que, infelizmente, continua a ser a base de nossos sistemas educacionais - inclusive na educação infantil. A busca de respostas que contribuam para vencer essas inadequações, concluem as autoras, deve necessariamente "incluir a perspectiva da criança em toda pesquisa sobre infância, brincadeira e educação infantil" (Lordelo et al., 2008, p. 137); dito de outra forma, superar efetivamente o mito do futurismo.

Há outros ângulos pelos quais esse mito pode ser questionado. $\mathrm{O}$ futurismo baseia-se em um modelo de causalidade e em procedimentos de pesquisa cuja insuficiência para o estudo do desenvolvimento tem sido repetidamente apontada e evidenciada. Thoman (1979) argumenta que é necessário caminhar na direção de uma ciência da infância mais humanística e mais holística, o que envolve reconhecer que: (a) resultados experimentais sobre aspectos isolados devem ser integrados em uma concepção de infância como sistema adaptativo; (b) estudos transversais de relações causais não são estudos de desenvolvimento; o desenvolvimento é um processo que ocorre no tempo; (c) a orientação tradicional da pesquisa é analítica, enquanto o processo de desenvolvimento é essencialmente integrativo, envolvendo síntese de determinantes altamente complexos; (d) a criança não é passiva nesse processo, e o desenvolvimento não ocorre no vácuo nem em laboratórios ou em contextos que os mimetizam; o percurso de desenvolvimento é singular em cada caso e portanto, no limite, imprevisível.

Assumindo o desenvolvimento como processo no tempo, Marková (1987) remonta ao princípio aristotélico da não contradição a origem das dificuldades da ciência ocidental para estudá-lo como tal. Devido à marca indelével de Aristóteles, o pensamento ocidental teria dificuldade de integrar a contribuição de pares de opostos, ou dicotomias (organismo versus ambiente, estabilidade versus instabilidade, conformidade versus inovação etc.) na explicação de processos psicológicos; ou seja, de substituir o princípio da não contradição, suficiente para o estudo de fenômenos estáticos, pela noção de Heráclito de "ser - não ser" simultâneos - um pensamento interacionista mais adequado para o estudo de processos.

O interacionismo pressupõe um modelo explicativo em que os termos em interação não podem ser dicotomicamente reduzidos a causa e efeito, mas no qual sua ação conjunta ou seus efeitos recíprocos promovem desenlaces com graus variáveis de liberdade (Carvalho, 1988b). $\mathrm{O}$ processo de desenvolvimento não é nem determinado nem totalmente indeterminado: é circunscrito (constrained), ou seja, delimitado e possibilitado em um campo de possibilidades previamente indeterminadas. $\mathrm{O}$ sentido de constraints contempla tanto o estreitamento de opções - ou caminhos irreversíveis, como 
proposto por Waddington (1956) em relação ao desenvolvimento de uma célula - como abertura de novas opções a partir de cada opção feita.

Explicitando essas asserções no caso da dicotomia organismo-ambiente (ou fatores genéticos-fatores ambientais), endêmica no estudo do desenvolvimento, "modelos dicotômicos são insuficientes para a compreensão da complexidade da interação meio-organismo que constitui a essência do processo de desenvolvimento" (Carvalho, 1987, p. 7). O ambiente provê as experiências que moldam o curso do desenvolvimento; mas, concomitantemente, o organismo, com seu repertório específico da espécie, seleciona ativamente certas formas de experiência e ignora outras, construindo dessa forma seu próprio ambiente (Hinde, 1974). Reduzido a uma linguagem causal simplificada, isso implica que nem organismo nem ambiente podem ser vistos como causas independentes dos desenlaces do processo de desenvolvimento, por mais que a psicologia do desenvolvimento tenha se esforçado para qualificar um ou outro como tais (Sameroff, 2010), hereditariedade e ambiente têm seu papel em tudo.

Sintetizando, o estudo do processo de desenvolvimento pede formas mais ricas de determinismo, incompatíveis com uma concepção futurista desse processo; requer a integração de uma visão biopsicossocial da criança desde o nascimento, que a reconheça em sua humanidade e em seu momento de vida; carece ainda de um reconhecimento de sistemas diádicos e poliádicos como unidades de recorte e de análise, ultrapassando o foco no indivíduo que é frequentemente característico de um determinismo linear e de procedimentos de intervenção.

Enquanto discurso, todas as asserções anteriores podem parecer consensuais na Psicologia do Desenvolvimento contemporânea. Serão de fato? Essas velhas questões estarão realmente resolvidas de maneiras que se reflitam nas práticas de pesquisa e, principalmente, nas de intervenção? Modelos conceituais mudam antes de metodologias e procedimentos para análise de dados, e a transformação de práticas de intervenção é ainda mais lenta, dada a dificuldade de trânsito comunicacional em redes sociais complexas - apesar da explosão contemporânea de sistemas e de velocidade de transmissão de informações. Por exemplo, não é raro encontrar a premissa de que é preciso o adulto (educador ou pesquisador) “dar voz e poder” à criança: será que a premissa significa que a criança não possui essas condições e que é preciso dotá-la para que as tenha? Ou será preciso encontrar meios de ouvir sua voz e enxergar o poder de que já é dotada? O significado ambíguo de "dar voz" e "dar poder" sugere que a premissa pode ser mal compreendida, conduzindo a práticas muito diversas. Essa própria ambiguidade desvela a recorrência da noção de que a criança é menos, e precisa ser ajudada para ser mais - dentro da compreensão adulta do que é ser mais. Da mesma forma, por detrás de discursos interacionistas e construtivistas é fácil reencontrar as dicotomias tradicionais entre organismo e ambiente, a dificuldade de integrar uma visão biopsicossocial do ser humano, como reivindicado por Morin já na década de 70 (Morin, 1979), e nela situar a infância.

Concluindo esta reflexão, sugerimos que, em geral, ainda é difícil encontrar questões novas relevantes no estudo contemporâneo do desenvolvimento, especialmente no que se refere ao mito do futurismo. O que encontramos é a recorrência das mesmas velhas questões, ainda não resolvidas apesar das transformações no discurso.

Para finalizar, arrisco apontar alguns caminhos que temos seguido em nosso trabalho (ver também, por exemplo, Carvalho et al., 1998) que nos parecem promissores. Já rejeitando os mitos do neonato incompetente, da neutralidade emocional e das competências aditivas, como compreender a criança como ser humano competente e adaptado desde a concepção? Por exemplo, observando-a em situações de seu cotidiano, que irão variar dependendo da idade, mas que sempre vão incluir outros seres humanos, 
adultos e/ou outras crianças de várias idades, parceiros potenciais de interação e, portanto, concebendo-a sempre como um ser social, parte de um sistema social; preferencialmente, observá-la em situações de atividade livre, sem interferência ou orientação de adultos; principalmente com crianças bem pequenas, que ainda não dominam o código verbal, dar atenção especial a seus mecanismos de comunicação e à sua eficácia (ou não) a cada momento; descrever a dinâmica interacional sem incorrer na tentação de atribuir à criança condições ainda ausentes, como intencionalidade, quando evidências dessas condições não emergem na própria dinâmica da interação.

O que é interação? A partir de registros videogravados em situações desse tipo, definimos interação como o potencial de regulação entre os membros de um grupo social. A ocorrência de regulação (que pode ser ou não recíproca) é identificada quando o comportamento de um (ou mais) parceiro(s) só pode ser entendido se o outro parceiro for levado em conta - por exemplo, em uma imitação não reciprocada, na orientação da atenção para o parceiro - condição mínima para a ocorrência de outras modalidades de regulação. Uma regulação recíproca ocorre quando a compreensão do comportamento de um ou mais parceiros requer levar em conta as ações de ambos (ou mais) e tem o potencial de gerar significados compartilhados por eles. Um significado compartilhado tem um potencial de persistência, ou seja, de ocorrer no momento seguinte ou em outro momento, seja com o mesmo ou com outro parceiro que passou a compartilhálo. Um significado compartilhado abreviado em um sinal caracteriza uma correlação, ou seja, a simplificação de um significado compartilhado, o que pode aumentar seu potencial de persistência. A duração da persistência não é relevante, e sim o próprio fato do compartilhamento recorrente (Carvalho et al., 1998).

O princípio de persistência de significados abre caminho para o reconhecimento e a compreensão de dois outros aspectos da sociabilidade humana, facilmente identificáveis em adultos, mas que já se manifestam na infância: a criação de cultura e a seletividade em relação a parceiros sociais. Persistência de significados está na raiz do conceito de cultura e permite compreender de que modo crianças ainda bem pequenas já criam cultura - a chamada peer culture (cultura de pares) observada e analisada por William Corsaro (2011). A seletividade em relação a parceiros sociais na infância tem sua expressão privilegiada na relação de apego, mas se manifesta também, sob formas e provavelmente com funções diferentes, nas relações de parcerias preferenciais, tanto em adultos (amizades, redes sociais) quanto entre pares de idade. Observando crianças de cerca de três anos brincando em díades de parceiros preferenciais e não preferenciais, definidos pela frequência de interações, verificamos que parceiros privilegiados já trazem para o campo interacional uma bagagem de significados compartilhados que permite que entrem facilmente em regulação recíproca e construam novos significados, tal como ocorre nas trocas sociais entre amigos na vida adulta (Carvalho \& Rubiano, 2004). A ocorrência precoce desses fenômenos característicos da sociabilidade humana evidencia e justifica a afirmação de que seres humanos são biologicamente socioculturais (Bussab \& Ribeiro, 1998).

A literatura psicológica relativa ao processo de desenvolvimento tem focalizado o vínculo em dois contextos teórico-empíricos principais: o apego mãe-filho ou adulto-criança, e a amizade na infância (Carvalho \& Rubiano, 2004). Nos dois casos, apesar de grandes diferenças de enfoque e de fundamentação teórica, as autoras identificam um "foco nas dimensões e funções individuais do fenômeno, dificultando a percepção e a consideração de suas dimensões e funções interpessoais e grupais e a análise do vínculo como fenômeno social humano" (p. 174). É esse foco que vimos tentando ultrapassar em nosso trabalho ao longo dos últimos vinte e cinco anos, buscando uma análise que reconheça a inseparabilidade dos planos do social e do individual e contemple as várias dimensões do conceito de social. 


\section{Referências}

Bergamasco, N. H. P. (1997). Expressão facial como acesso à consciência do recém-nascido. Psicologia -USP, 8(2), 275-286. https://www. revistas.usp.br/psicousp/issue/view/8168/456

Bergamasco, N. H. P., \& Beraldo, K. E. A. (1990). Facial expressions of neonate infants in response to gustatory stimuli. Brazilian Journal of Medical and Biological Research, 23(3-4), 245249. https://pubmed.ncbi.nlm.nih.gov/2094539/

Bruner, J. S. (1976). Nature and uses of immaturity. In J. S. Bruner, A. Jolly, \& K. Sylva (Eds.), Play: Its Role in Development and Evolution (pp. 28-65). Penguin Books.

Bussab, V. S. R., \& Ribeiro, F. J. L. (1998). Biologicamente social. In L. Souza, M. F. Q. Freitas, \& M. M. P. Rodrigues (Orgs.), Psicologia: Reflexões (im)pertinentes (pp. 175194). Casa do Psicólogo.

Bussab, V. S. R., Pedrosa, M. I., \& Carvalho, A. M. A. (2007). Encontros com o outro: Empatia e intersubjetividade no primeiro ano de vida. Psicologia USP, 18(2), 99-132. https://www.revistas.usp.br/psicousp/article/ view/41923/45591

Carvalho, A. M. A. (1983). O desenvolvimento da criança. Pediatria Moderna, 18(5), 269-280. Id: lil-20055

Carvalho, A. M. A. (1987). O estudo do desenvolvimento. Psicologia, 13, 1-13.

Carvalho, A. M. A. (1988a). Etologia das relações mãe-criança no ser humano. Anais do VI Encontro Anual de Etologia (pp. 39-45). Florianópolis, SC.

Carvalho, A. M. A. (1988b). Algumas reflexões sobre o uso da categoria "interação social". Anais da XVII Reunião Anual de Psicologia da SPRP (pp. 511-515). Ribeirão Preto, SP.

Carvalho, A. M. A., Império-Hamburger, A., \& Pedrosa, M. I. (1998). Interaction, regulation and correlation in the context of human development: Conceptual discussion and empirical examples. In M. C. D. P. Lyra \& J. Valsiner (Eds.), Construction of psychological processes in interpersonal communication (pp. 155-180). Ablex Publishing Corporation.

Carvalho, A. M. A., \& Rubiano, M. R. B. (2004). Vínculo e compartilhamento na brincadeira de crianças. In M. C. Rosseti-Ferreira, K. S.
Amorim, A. P. S. S. Silva, \& A. M. A. Carvalho (Orgs.), Rede de significações e o estudo do desenvolvimento humano (pp. 171-187). ArtMed.

Corsaro, W. A. (2011). Culturas de pares de crianças e reprodução interpretativa. In W. A. Corsaro, Sociologia da infância (pp. 127-152). ArtMed.

Delevati, N. M., \& Bergamasco, N. H. P. (1999). Pain in the neonate: An analysis of facial movements and crying in response to nociceptive stimuli. Infant Behavior and Development, 22(1), 139-145. https://doi.org/10.1016/S01636383(99)80011-3

Hinde, R. A. (1974). Biological bases of Human Social Behaviour. McGraw Hill.

Houaiss, A., \& Villar, M. S. (2001). Mito. In Dicionário Houaiss da língua portuguesa (p. 1.936).

Lordelo, E. R., Carvalho, A. M. A., \& Bichara, I. D. (2008). Infância roubada brincadeira e educação infantil no Brasil. In L. V. C. Moreira \& A. M. A. Carvalho (Orgs.), Família e Educação (pp. 119-138). Paulinas.

Marková, I. (1987). On the interaction of opposites in psychological processes. Journal for the Theory of Social Behaviour, 17(3), 279-299. https://doi. org/10.1111/j.1468-5914.1987.tb00100.x

Morin, E. (1979). O enigma do homem: Para uma nova Antropologia. Zahar.

Sameroff, A. (2010). A unified theory of development: A dialectic integration of nature and nurture. Child Development, 81(1), 6-22. https://doi. org/10.1111/ j.1467-8624.2009.01378.x

Seidl de Moura, M. L., \& Ribas, A. F. P. (2004). Evidências sobre características de bebês recémnascidos: Um convite a reflexões teóricas. In $\mathrm{M}$. L. Seidl de Moura (Org.), O bebê do século XXI e a Psicologia em desenvolvimento (pp. 22-59). Casa do Psicólogo.

Thoman, E. (1979). Changing views on the being and becoming of infants (Nantucket Conference). In E. Thoman (Ed.), Origins of the infant's social responsiveness. Wiley.

Tudella, E., Oishi, J., \& Bergamasco, N. H. P. (2000). The effect of oral-gustatory, tactile-bucal and tactile-manual stimulation on the behavior of the hands in newborns. Developmental Psychobiology, 37(2), 82-89. https://doi. org/10.1002/1098-2302(200009)37:23.0.CO; 2-B 
Waddington, C. H. (1956). Principles of Embriology.

Recebido: 05/06/2021

In S. F. Gilbert (Ed.), A conceptual history of

$1^{a}$ revisão: 07/07/2021

modern embriology (pp. 181-206). Plenum

Aceite final: 30/07/2021

Press.

\section{Apêndice}

\section{Destaques de Referências para Aprofundamento no Tema}

Bruner, J. S. (1976). Nature and uses of immaturity. In J. S. Bruner, A. Jolly, \& K. Sylva (Eds.), Play: Its Role in Development and Evolution (pp. 28-65). Penguin Books.

O autor apresenta argumentos e evidências que apontam as vantagens adaptativas da imaturidade na infância.

Thoman, E. (1979). Changing views on the being and becoming of infants (Nantucket Conference). In E. Thoman (Ed.), Origins of the infant's social responsiveness. Wiley.

A autora discute pressupostos da Psicologia do Desenvolvimento e necessidade de integrar resultados para conceber a infância como sistema adaptativo.

\section{Sugestões de Leituras}

Bruner, J. S., Jolly, A., \& Sylva, K. (Eds.). (2017). Play: Its role in development and evolution. https://www. researchgate.net/publication/328486788_Play_its_role_in_development_and_evolution

Carvalho, A. M. A., Pedrosa, M. I., \& Rossetti-Ferreira, M. C. (2012). Aprendendo com a criança de zero a seis anos. Cortez Editora.

Carvalho, A. M. A., \& Pedrosa, M. I. (Orgs.). (2020). Física e psicologia: Um ensaio de interdisciplinaridade. Edicon.

(cc)BY BY O(s) autor(es), 2021. Acesso aberto. Este artigo está distribuído nos termos da Licença Internacional Creative Commons Atribuição 4.0 (http://creativecommons.org/licenses/by/4.0/), que permite o uso, distribuição e reprodução sem restrições em qualquer meio, desde que você dê crédito apropriado ao(s) autor(es) original(ais) e à fonte, fornecer um link para a licença Creative Commons e indicar se as alterações foram feitas. 